\title{
Characteristics of HER2 FISH-Equivocal Breast Cancers and the Impacts on HER2 Status of 2018 ASCO/CAP Guideline
}

Hui Kong

Zhongshan Hospital Fudan University https://orcid.org/0000-0001-9158-8396

Qianming Bai

Fudan University Shanghai Cancer Center

Anqi Li

Fudan University Shanghai Cancer Center

Xiaoyan Zhou

Fudan University Shanghai Cancer Center

Wentao Yang ( $\nabla$ yangwt2000@163.com )

Fudan University Shanghai Cancer Center https://orcid.org/0000-0001-7712-822X

Research

Keywords: Breast cancer, HER2 status, 2018 ASCO/CAP

Posted Date: February 19th, 2021

DOI: https://doi.org/10.21203/rs.3.rs-248067/v1

License: (c) (1) This work is licensed under a Creative Commons Attribution 4.0 International License. Read Full License 


\section{Abstract}

Background: According to 2018 ASCO/CAP guideline, HER2 FISH-equivocal breast cancers will be categorized as HER2 negative except those with HER2 IHC 3+. However, whether or not HER2 FISHequivocal breast cancers was a heterogeneous group has not been well illustrated.

Methods: 195 HER2 FISH-equivocal breast cancers were collected between 2014 and 2018. The molecular subtype was determined according to 2013 St Gallen consensus, and HER2 status was also redefined following 2018 ASCO/CAP guideline. All cases were classified into 4 groups according to the average HER2 copy number (4.0-4.4, 4.5-4.9, 5.0-5.4, 5.5-5.9 signals/cell). The relationship between HER2 copy number and clinicopathological parameters was analyzed.

Results: 183 (93.8\%) of 195 FISH-equivocal cases were of luminal subtype, while the other $12(6.2 \%)$ were undetermined. Using 2018 ASCO/CAP guideline, all FISH-equivocal cases were recategorized to HER2 negative. Therefore, 31(15.9\%) cases were luminal A-like, 152 (77.9\%) were luminal B-like (HER2 negative) and $12(6.2 \%)$ were triple negative. The average HER2 copy number showed positive correlation with chromosome 17 polysomy, but had no significant association with other clinicopathological parameters as well as prognosis. $17(8.7 \%)$ cases were treated with trastuzumab, but showed no difference in prognosis with patients who didn't receive targeted therapy.

Conclusions: In this study, all HER2 FISH-equivocal breast cancers were reclassified to be HER2 negative according to 2018 ASCO/CAP guideline. Most of these patients were luminal B-like (HER2 negative). The average HER2 copy number had no significant association with clinicopathological parameters, as well as prognosis.

\section{Background}

Breast cancer remains the leading cause of cancer incidence and mortality in women worldwide. Global cancer statistics estimated that there was about 2.1 million new cases in 2018 , accounting for almost $25 \%$ of all female cancers cases [1].

Human epidermal growth factor receptor 2 (HER2) is found to be involved in several key cellular signal pathways, including proliferation, migration, and adhesion in breast cancers [2]. HER2-targeted drugs, such as Trastuzumab, which inhibit downstream signaling of these pathways, are very effective for HER2 positive breast cancer patients. Therefore, HER2 status is crucial in choosing therapeutic scheme. Currently, HER2 testing mainly in two ways, immunohistochemistry (IHC) assays for protein overexpression and fluorescence in situ hybridization (FISH) for gene amplification. Although the commonly adopted routine is to perform IHC assays initially, HER2 FISH is required when the IHC result is equivocal. HER2 status was determined according to the American Society of Clinical Oncology/College of American Pathologists (ASCO/CAP) guideline recommendations [3-5]. 
However, 2013 ASCO/CAP guideline caused an increased HER2 equivocal cases [6-8], whose HER2 status is undefined. It is hard for medical oncologists to decide whether or not using HER2-targeted therapy for equivocal cases. In 2018, the guideline was updated and recommended a definitive diagnosis for the former HER2 FISH-equivocal breast cancers, and almost all equivocal cases were categorized as HER2 negative except those with HER2 IHC 3+ [5]. Since the dual-probe FISH test gives an exact score of average HER2 copy number and the value ranges from 4.0 to 6.0 . However, whether or not HER2 FISHequivocal breast cancers could be a heterogeneous group according to different HER2 copy number has not been well investigated.

In this study, we collected 195 HER2 FISH-equivocal invasive breast cancers diagnosed following 2013 ASCO/CAP guideline [4], and redefined HER2 status according to 2018 updated guideline. The molecular subtype of those cases were categorized basing on different HER2 status. The relationship between HER2 copy number and clinicopathological parameters was analyzed. The impact of different HER2 copy number on prognosis was also illustrated.

\section{Methods}

\section{Patients and study design}

According to the 2013 ASCO/CAP guideline, 195 invasive breast cancers diagnosed to be HER2 FISHequivocal from 2014 to 2018 were retrieved from Shanghai Cancer Center. For all cases, IHC and FISH assays were performed on the same tissue block of surgical specimen. The diagnoses were reviewed by two experienced senior pathologists. Clinical characteristics of 195 study subjects are presented in Table 1. 
Table 1

Characteristics of study subjects.

\begin{tabular}{|c|c|c|}
\hline & Variable & $\mathrm{n}(\%)$ \\
\hline Age, yr & Median (range) & $53(28-82)$ \\
\hline \multicolumn{3}{|c|}{ Histologic subtype } \\
\hline & Ductal, NOS & $181(92.8)$ \\
\hline & Lobular & $4(2.1)$ \\
\hline & Micropapillary & $9(4.6)$ \\
\hline & Solid papillary with invasion & $1(0.5)$ \\
\hline \multicolumn{3}{|c|}{ Histologic grade } \\
\hline & I & $2(1.0)$ \\
\hline & II & $94(48.2)$ \\
\hline & III & $99(50.8)$ \\
\hline \multicolumn{3}{|l|}{ ER status } \\
\hline & ER+ & $183(93.8)$ \\
\hline & ER- & $12(6.2)$ \\
\hline \multicolumn{3}{|l|}{ PR status } \\
\hline & PR+ & $160(82.1)$ \\
\hline & PR- & $35(17.9)$ \\
\hline \multirow[t]{5}{*}{ HER2 status } & IHC status & \\
\hline & $1+$ & $10(5.1)$ \\
\hline & $2+$ & 185 (94.9) \\
\hline & Dual-probe ISH status & \\
\hline & \pm & $195(100.0)$ \\
\hline \multicolumn{3}{|l|}{ Polysomy 17} \\
\hline & Yes & $137(70.3)$ \\
\hline & No & $58(29.7)$ \\
\hline \multicolumn{3}{|l|}{ Ki-67 } \\
\hline & $凶 20 \%$ & $49(25.1)$ \\
\hline
\end{tabular}




\begin{tabular}{|c|c|c|}
\hline & Variable & $\mathrm{n}(\%)$ \\
\hline & $\geq 20 \%$ & $146(74.9)$ \\
\hline TNM stage & \multicolumn{2}{|l|}{$\mathrm{T}$ (Primary tumour) } \\
\hline & $\mathrm{T} 1$ & $93(47.7)$ \\
\hline & T2 & $98(50.3)$ \\
\hline & T3 & $1(0.5)$ \\
\hline & T4 & $3(1.5)$ \\
\hline & \multicolumn{2}{|c|}{ N (Regional lymph nodes) } \\
\hline & NO & $104(53.3)$ \\
\hline & N1 & $57(29.2)$ \\
\hline & N2 & $19(9.8)$ \\
\hline & N3 & $15(7.7)$ \\
\hline & \multicolumn{2}{|c|}{ M (Distant metastasis) } \\
\hline & MO & $192(98.5)$ \\
\hline & M1 & $3(1.5)$ \\
\hline \multicolumn{3}{|l|}{ Treatment } \\
\hline & Chemotherapy & 159 (81.5) \\
\hline & Radiotherapy & $89(45.6)$ \\
\hline & Hormonal therapy & $123(63.1)$ \\
\hline & Trastuzumab & $17(8.7)$ \\
\hline
\end{tabular}

HER2 status was re-determined following the 2018 ASCO/CAP guideline. Molecular subtype was determined according to the 2013 St Gallen consensus [9]. We divided the 195 individuals into 4 groups according to the average HER2 copy number (4.0-4.4, 4.5-4.9, 5.0-5.4, 5.5-5.9 signals/cell, respectively). Clinicopathological factors, such as histological subtype and grade, ER and PR status, chromosome 17 polysomy and so on, were investigated among 4 groups to find the intrinsic association with HER2 copy number.

\section{Ihc And Fish}

IHC staining was performed using the Ventana Bench Mark ultra autostainer and the Ventana Ultra View universal DAB detection kit (Ventana Medical System Inc, Roche Tucson, Arizona, USA). The primary 
antibodies included ER, PR, HER2, Ki-67, E-cadherin, p120, AE1/AE3, calponin and p63. All of these antibodies were from Roche Ventana. Appropriate positive and negative controls were carried out simultaneously for all stains.

The status of ER and PR were determined following the criteria of ASCO/CAP guideline [10]. The ratio of nuclei-positive tumor cells to all tumor cells calculated visually represented the $\mathrm{Ki}-67$ value, and a high $\mathrm{Ki}-$ 67 expression was defined as $\geq 20 \%$ [9]. E-cadherin and p120 were employed to discriminate lobular carcinomas from ductal carcinomas. Double immunostainings were performed with AE1/AE3/P63 or $\mathrm{AE} 1 / \mathrm{AE} 3 /$ calponin to confirm the presence of invasive component.

A dual-probe FISH test using the PathVysion HER2 DNA probe Kit (Vysis Inc, Downers Grove, IL) was performed on the same specimen as IHC test following the manufacturer's instructions. FISH results were interpreted by two experienced pathologists independently.

\section{Follow-up Information}

Patients follow-up were carried out in Shanghai Cancer Center. The overall survival (OS) was computed from the date of surgery till death of any cause. Disease-free survival (DFS) was calculated from the date of surgery till the first proven event including recurrence, distant metastasis, second primary tumor and death of any cause. The last follow-up was conducted in August 2018.

\section{Statistical analysis}

The correlation between clinicopathological factors and the average HER2 copy number was analyzed by chi-squared test or Fisher's exact test. OS and DFS were estimated with Kaplan-Meier curves analysis. IBM SPSS Statistics software (version 21) was used to perform the statistical analysis. All $P$ values were two-tailed, and difference with $P$ value $<0.05$ was considered to be statistically significant.

\section{Results}

\section{Histologic subtype and grade}

With regard to histologic subtype, 181 (92.8\%) of the 195 HER2 FISH-equivocal cases were invasive ductal carcinoma, not otherwise specified (NOS). In the rest 14 cases, 4 (2.1\%) cases were invasive lobular carcinoma, and $9(4.6 \%)$ cases were invasive micropapillary carcinoma, and only $1(0.5 \%)$ case was solid papillary with invasion.

In term of histological grade, the collected samples were mainly distributed between Grade II and Grade III. The numbers of corresponding cases were 94 (48.2\%) and 99 (50.8\%), respectively. Only $2(1 \%)$ cases were diagnosed as Grade I. 


\section{Her2 Status}

IHC and dual-probe FISH assays tests were both performed to determine HER2 status. IHC test results showed that $10(5.1 \%)$ of 195 cases were $1+$, and the rest $185(94.9 \%)$ cases were $2+$, and none was $3+$. In other words, 10 cases were negative and the rest were equivocal in term of HER2 status.

The dual-probe FISH assays results showed that 195 (100\%) cases were FISH equivocal according to 2013 ASCO/CAP guideline. After re-determined following the 2018 guideline, all the cases turned to be HER2 negative.

\section{Molecular Subtype}

In this study, the molecular subtype was determined in accordance with 2013 St Gallen International Expert consensus, which mainly took ER, PR, HER2 and Ki-67 as indicators.

The rates of ER positive and PR positive were 93.8\% (183/195) and 82.1\% (160/195) respectively. The Ki67 status of $146(74.9 \%)$ cases was high, and 49 cases (25.1\%) were low, when the cutoff value was set to be $20 \%$. The HER2 status of samples were different according to 2013 or 2018 ASCO/CAP guidelines as described previously.

As indicated in Fig. 1, there were 59 luminal subtype and 4 undetermined in group A, 65 luminal subtype and 2 undetermined in group $B, 45$ luminal subtype and 5 undetermined in group $C, 14$ luminal subtype and 1 undetermined in group D. In summary, 183 (93.8\%) of 195 HER2 FISH-equivocal cases were luminal subtype, while the other 12 (6.2\%) were undetermined according to 2013 ASCO/CAP guideline. After HER2 status was re-determined following 2018 ASCO/CAP guideline, all the undetermined cases became triple negative subtype, while the luminal subtype could be further categorized into luminal A-like and luminal B-like (HER2 negative) subtypes. In summary, 31(15.9\%) cases were luminal A-like, and 152 (77.9\%) were luminal B-like (HER2 negative) and 12 (6.2\%) were triple negative.

\section{Clinicopathological Parameters And The Relationship With 4 Groups}

In this study, 195 HER2 FISH-equivocal samples were divided into 4 groups according to the average HER2 copy number (4.0-4.4, 4.5-4.9, 5.0-5.4, 5.5-5.9 signals/cell, respectively). The number of cases in each group were $63,67,50$ and 15 , respectively (Table 2). The principal clinicopathological parameters investigated were patients ages, histological subtypes, histological grades, ER status, PR status, and HER2 status, the presence of chromosome 17 polysomy, Ki-67 status and TNM stage. 
Table 2

Correlations of average HER 2 copy number and major clinicopathological factors.

\begin{tabular}{|c|c|c|c|c|c|}
\hline \multirow[t]{2}{*}{ Characteristics } & \multicolumn{4}{|c|}{ Average $H E R 2$ copy number } & \multirow[t]{2}{*}{$P$ value } \\
\hline & 4.0-4.4 & $4.5-4.9$ & $5.0-5.4$ & $5.5-5.9$ & \\
\hline \multicolumn{5}{|l|}{ Age (year) } & 0.15 \\
\hline$\leq 53$ & $32(50.8)$ & $41(61.2)$ & $20(40.0)$ & $5(33.3)$ & \\
\hline$凶 53$ & $31(49.2)$ & $26(38.8)$ & $30(60.0)$ & $10(66.7)$ & \\
\hline \multicolumn{5}{|l|}{ Histopathology } & 0.969 \\
\hline IDC* & $58(92.1)$ & $62(92.5)$ & $49(98.0)$ & $12(80.0)$ & \\
\hline Non-IDC & $5(7.9)$ & $5(7.5)$ & $1(2.0)$ & $3(20.0)$ & \\
\hline \multicolumn{5}{|l|}{ Histologic grade } & 0.129 \\
\hline I & $0(0.0)$ & $0(0.0)$ & $2(4.0)$ & $0(0.0)$ & \\
\hline II & $27(42.9)$ & $34(50.7)$ & $23(46.0)$ & $10(66.7)$ & \\
\hline III & $36(57.1)$ & $33(49.3)$ & $25(50.0)$ & $5(33.3)$ & \\
\hline \multicolumn{5}{|l|}{ ER } & 0.538 \\
\hline Positive & $59(93.7)$ & $65(97.0)$ & $45(90.0)$ & $14(93.3)$ & \\
\hline Negative & $4(6.3)$ & $2(3.0)$ & $5(10.0)$ & $1(6.7)$ & \\
\hline \multicolumn{5}{|l|}{ PR } & 0.257 \\
\hline Positive & $48(76.2)$ & $57(85.1)$ & $43(86.0)$ & $12(80.0)$ & \\
\hline Negative & $15(23.8)$ & $10(14.9)$ & $7(14.0)$ & $3(20.0)$ & \\
\hline \multicolumn{5}{|l|}{ Ki-67 } & 0.065 \\
\hline$\nabla 20 \%$ & 19 (30.2) & $20(29.9)$ & $7(14.0)$ & $3(20.0)$ & \\
\hline$\geq 20 \%$ & $44(69.8)$ & $47(70.1)$ & $43(86.0)$ & $12(80.0)$ & \\
\hline \multicolumn{5}{|l|}{ Polysomy 17} & \0.001** \\
\hline Yes & $30(47.6)$ & $48(71.6)$ & $46(92.0)$ & $13(86.7)$ & \\
\hline No & $33(52.4)$ & $19(28.4)$ & $4(8.0)$ & $2(13.3)$ & \\
\hline \multicolumn{5}{|c|}{ Primary Tumor $(\mathrm{T})$} & 0.776 \\
\hline T1 & $31(49.2)$ & $33(49.3)$ & $21(42.0)$ & $8(53.3)$ & \\
\hline \multicolumn{6}{|l|}{ Abbreviations: } \\
\hline *: Invasive breas & cancer & & & & \\
\hline
\end{tabular}




\begin{tabular}{|c|c|c|c|c|c|}
\hline \multirow[t]{2}{*}{ Characteristics } & \multicolumn{4}{|c|}{ Average $H E R 2$ copy number } & \multirow[t]{2}{*}{$P$ value } \\
\hline & $4.0-4.4$ & $4.5-4.9$ & $5.0-5.4$ & $5.5-5.9$ & \\
\hline T2 & $30(47.6)$ & $33(49.3)$ & $28(56.0)$ & $7(46.7)$ & \\
\hline T3 & $0(0.0)$ & $1(1.5)$ & $0(0.0)$ & $0(0.0)$ & \\
\hline T4 & $2(3.2)$ & $0(0.0)$ & $1(2.0)$ & $0(0.0)$ & \\
\hline \multicolumn{5}{|c|}{ Regional Lymph Nodes (N) } & 0.806 \\
\hline NO & $32(50.8)$ & $40(59.7)$ & $25(50.0)$ & $7(46.7)$ & \\
\hline N1 & $20(31.7)$ & $15(22.4)$ & $17(34.0)$ & $5(33.3)$ & \\
\hline N2 & $8(12.7)$ & $6(9.0)$ & $3(6.0)$ & $2(13.3)$ & \\
\hline N3 & $3(4.8)$ & $6(9.0)$ & $5(10.0)$ & $1(6.7)$ & \\
\hline \multicolumn{5}{|c|}{ Distant Metastasis (M) } & 0.804 \\
\hline MO & $62(98.4)$ & $66(98.5)$ & $50(100.0)$ & $14(93.3)$ & \\
\hline M1 & $1(1.6)$ & $1(1.5)$ & $0(0.0)$ & $1(6.7)$ & \\
\hline \multicolumn{6}{|l|}{ Abbreviations: } \\
\hline \multicolumn{6}{|c|}{ *: Invasive breast cancer } \\
\hline
\end{tabular}

The correlation between those parameters and average HER2 copy number were analyzed. The results suggested that the average HER2 copy number showed positive correlation with the presence of chromosome 17 polysomy $(P<0.001)$. However, there were no significant correlation with other clinicopathological parameters.

\section{Prognosis}

The individualized therapy was decided through a multidisciplinary team meeting. All involved patients have taken surgery and treated with different combinations of chemotherapy, radiotherapy and hormonal therapy. In addition, among the 195 HER2 FISH-equivocal individuals, 17 (8.7\%) have taken targeted therapy of trastuzumab preoperatively or postoperatively.

Patients follow-up data was collected in a long term and the most recent data was collected in August 2018. Among 195 study subjects, 16 patients who were out of contact, were excluded from survival analysis. The median duration of follow-up was 34 months (range 10-55). By the end of data collection, no recurrence of breast cancer had been seen, while distant metastasis had occurred in five cases, including one lung metastasis, one liver metastasis, and three bone metastases. Besides, four individuals died without detail information. 
Treatment response and prognosis of 17 patients taking HER2 targeted therapy were the major problem we concerned. Although two of them were treated with trastuzumab before surgery, they also received chemotherapy and/or radiotherapy. The Miller-Payne grading system was used to assess pathologic response to neoadjuvant chemotherapy [11], and the two patients were categorized as grade 3 and grade 4. The other 15 patients took targeted therapy after surgery, and no recurrence had been seen by the end of the follow-up.

Univariate analysis of the impact of prognostic factors on DFS and OS of breast cancer patients was performed. The results showed that the $\mathrm{N}$ (regional lymph nodes) stage significantly affected OS, and the M (distant metastasis) stage significantly affected both DFS and OS. However, the average HER2 copy number in FISH test had no significant impact on DFS and OS of breast cancer patients. Meanwhile, DFS and OS showed no significant difference between patients with and without targeted therapy (Table 3 ).

Table 3

Univariate analysis of prognostic factors affecting DFS and OS in HER2 FISH-equivocal tumours.

\begin{tabular}{|c|c|c|c|}
\hline & & DFS & os \\
\hline Factors & Subset & $P$ value & $P$ value \\
\hline Age (year) & 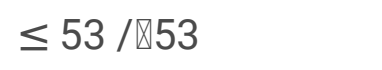 & 0.299 & 0.059 \\
\hline Histologic subtype & IDC§ / Non-IDC & 0.917 & 0.978 \\
\hline Histologic grade & I / II / III & 0.888 & 0.967 \\
\hline ER & Positive / Negative & 0.425 & 0.531 \\
\hline PR & Positive / Negative & 0.539 & 0.146 \\
\hline HER2 IHC score & $1+/ 2+$ & 0.518 & 0.643 \\
\hline Average HER2 copy number & $1 / 2 / 3 / 4$ & 0.897 & 0.887 \\
\hline Ki-67 & $\nabla 20 \% / \geq 20 \%$ & 0.674 & 0.167 \\
\hline Target therapy & Yes / No & 0.439 & 0.582 \\
\hline T stage & T1 / T2 / T3 / T4 & 0.965 & NA \\
\hline N stage & N0 / N1 / N2 / T3 & 0.146 & $0.009 *$ \\
\hline M stage & $\mathrm{M} 0 / \mathrm{M} 1$ & $0.003^{*}$ & $0.000 *$ \\
\hline \multicolumn{4}{|l|}{ Abbreviations: } \\
\hline \multicolumn{4}{|l|}{ §: Invasive breast cancer } \\
\hline 9: Four groups according to a & copy number: 4.0-4. & $5.0-5.4,5$ & signals \\
\hline
\end{tabular}




\section{Discussion}

HER2-positive breast cancers are eligible for HER2-targeted therapy, such as trastuzumab and lapatinib $[12,13]$. It is just what makes the determination of HER2 status a routine pathological examination for breast cancer patients worldwide [14].

$\mathrm{IHC}$ assay is performed for every breast cancer patient as routine, while FISH is an optional test. However, HER2 FISH is required when the IHC results is $2+$ according to the ASCO/CAP guideline. Unfortunately, the result still can be equivocal even in HER2 FISH test, if the ratio of HER2 to CEP17 is $<2.0$ with an average HER2 copy number between 4.0 and 6.0 [4]. It is hard for medical oncologists to make treatment decision for FISH equivocal patients. Therefore, an Expert Panel updated ASCO/CAP guideline to clarify HER2 equivocal status in 2018.

According to the new guideline, HER2 FISH-equivocal breast cancers will be categorized as HER2 negative except those with HER2 IHC 3+. As such, HER2 targeted therapy was not recommended to those patients. Our results indicated that HER2 copy number was related with chromosome 17 polysomy, but had no impact on prognosis. When it comes to the molecular subtype, the majority of HER2 FISH-equivocal breast cancers turned into luminal B-like (HER2 negative). Those results were consistent with other studies.

Similarly, in our study, 195 FISH equivocal breast cancers consisted of 10 (5.1\%) IHC $1+$ and 185 (94.9\%) IHC 2+, and there was no case could get an IHC 3+. As such, all of the 195 equivocal cases were recategorized to HER2 negative, thus those patients would not be treated with HER2 targeted therapy. Apparently, HER2 FISH-equivocal breast cancers was a heterogeneous group due to different HER2 copy number between 4.0 and 6.0. To investigate what factors were the average HER2 copy number associated with, we analyzed the clinicopathological factors, molecular subtypes and prognosis between 4 groups. Our results indicated that different HER2 copy number was related with chromosome 17 polysomy, but had no impact on prognosis. When it comes to the molecular subtype, discriminate the molecular subtype of breast cancers could help us to understand the different clinical characteristics, treatment response and prognosis. HER2 FISH-equivocal breast cancers transferred to luminal B-like (HER2 negative) from undetermined subtype. Those results were consistent with other studies. Guo et al. demonstrated that HER2 equivocal cases according to the 2013 ASCO/CAP guideline showed similar biological behavior with luminal B type tumors [15]. Besides, Tong et al. reported similar clinicopathological features and mRNA expression levels between HER2 equivocal and HER2 negative cases. Furthermore, they pointed out that these two subsets showed comparable survival outcome in the absence of HER2 targeted therapy [16]. Those findings were also shown in previous researches [17, 18]. In this study, 17 HER2 FISH-equivocal individuals took targeted therapy preoperatively or postoperatively. Long term follow-up is necessary to find out whether this group of patients can benefit from HER2 targeted therapy or not.

In this study, chromosome 17 polysomy showed positive correlation with average HER2 copy number. Actually, polysomy 17 is a major cause of HER2 FISH equivocal [19]. Generally, breast cancer patients 
with polysomy 17 have not been considered eligible for HER2 targeted therapy. Nevertheless, researches indicated that complete chromosome 17 polysomy is rare. Patients with polysomy 17 have high possibility to be HER2 positive with concomitant amplification of the centromeric region [20, 21]. Further investigation is needed to confirm whether polysomy 17 breast cancers benefit from HER2 targeted therapy.

\section{Conclusions}

In conclusion, our study indicated that all HER2 FISH-equivocal breast cancers were reclassified to be HER2 negative according to 2018 ASCO/CAP guideline. Most of these patients were luminal B-like (HER2 negative). The average HER2 copy number was positive correlated with the presence of polysomy 17 and had no significant impact on DFS and OS of breast cancer patients. Besides, DFS and OS showed no difference between patients with and without targeted therapy. Further underlying molecular mechanism study with long term follow-up is necessary for this special group of patients.

\section{Abbreviations}

ASCO/CAP

American Society of Clinical Oncology/College of American Pathologists

HER2

Human epidermal growth factor receptor 2

FISH

in situ hybridization

$\mathrm{IHC}$

immunohistochemistry

OS

overall survival

DFS

Disease-free survival

NOS

not otherwise specified

\section{Declarations}

\section{Ethics statement}

This study has been approved by the Institutional Review Board of Shanghai Cancer Center in Fudan University and carried out in accordance with the Declaration of Helsinki. Written informed consent was obtained from involved patients.

\section{Consent for publication}


Not applicable.

\section{Availability of data and materials}

The datasets used and/or analysed during the current study are available from the corresponding author on reasonable request.

\section{Competing interests}

The authors have no conflicts of interest to declare.

\section{Funding}

This work was supported by the Nature Science Foundation of Shanghai (grant number 18ZR1407600).

\section{Authors' contributions}

HK collected and analyzed the data and prepared the draft of manuscript. HK, QB and AL independently reviewed molecular subtype and HER2 status. XZ and WY conceived the idea, supervised all research and revised the manuscript. All authors have read and approved the final version of this manuscript.

\section{Acknowledgements}

Not applicable.

\section{References}

1. Bray F, Ferlay J, Soerjomataram I, Siegel RL, Torre LA, Jemal A. Global cancer statistics 2018: GLOBOCAN estimates of incidence and mortality worldwide for 36 cancers in 185 countries. Cancer J Clin. 2018;49(suppl 8):509. doi:10.3322/caac.21492.

2. Gutierrez C, Schiff R. HER 2: Biology, Detection, and Clinical Implications. Arch Pathol Lab Med. 2011;135(1):55-62.

3. Wolff AC, Hammond MEH, Schwartz JN, et al. American Society of Clinical Oncology/College of American Pathologists guideline recommendations for human epidermal growth factor receptor 2 testing in breast cancer. JCO. 2007;25(1):118-45. doi:10.1200/JC0.2006.09.2775.

4. Wolff AC, Hammond MEH, Hicks DG, et al. Recommendations for human epidermal growth factor receptor 2 testing in breast cancer: American Society of Clinical Oncology/College of American Pathologists clinical practice guideline update. J Clin Oncol. 2013;31(31):3997-4013. doi:10.1200/JC0.2013.50.9984.

5. Wolff AC, Hammond MEH, Allison KH, et al. Human Epidermal Growth Factor Receptor 2 Testing in Breast Cancer: American Society of Clinical Oncology/College of American Pathologists Clinical Practice Guideline Focused Update. JCO. 2018: JCO.2018.77.873. doi:10.1200/JCO.2018.77.8738. 
6. Guo L, Yuan P, Zhang J, et al. Analysis of molecular subtypes for the increased HER2 equivocal cases caused by application of the updated 2013 ASCO/CAP HER2 testing guidelines in breast cancer. Breast Cancer Res Treat. 2017;166(1):77-84.

7. Hanna WM, Slodkowska E, Lu F-I, Nafisi H, Nofech-Mozes S. Comparative Analysis of Human Epidermal Growth Factor Receptor 2 Testing in Breast Cancer According to 2007 and 2013 American Society of Clinical Oncology/College of American Pathologists Guideline Recommendations. JCO. 2017;35(26):3039-45. doi:10.1200/JC0.2016.70.5319.

8. Bethune GC, Zanten DVV, Maclntosh RF, et al. Impact of the 2013 American Society of Clinical Oncology/College of American Pathologists guideline recommendations for human epidermal growth factor receptor 2 (HER2) testing of invasive breast carcinoma: a focus on tumours assessed as "equivocal" for HER2 gene amplification by fluorescence in-situ hybridization. Histopathology. 2015;67(6):880-7.

9. Goldhirsch A, Winer EP, Coates AS, et al. Personalizing the treatment of women with early breast cancer: highlights of the St Gallen International Expert Consensus on the Primary Therapy of Early Breast Cancer 2013. Ann Oncol. 2013;24(9):2206-23. doi:10.1093/annonc/mdt303.

10. Hammond MEH, Hayes DF, Dowsett M, et al. American Society of Clinical Oncology/College Of American Pathologists guideline recommendations for immunohistochemical testing of estrogen and progesterone receptors in breast cancer. J Clin Oncol. 2010;28(16):2784-95. doi:10.1200/JC0.2009.25.6529.

11. A new histological. grading system to assess response of breast cancers to primary chemotherapy: prognostic significance and survival. 2003;12(5):320-327. doi:10.1016/s0960-9776(03)00106-1.

12. Lapatinib or Trastuzumab Plus Taxane Therapy for Human Epidermal Growth Factor Receptor. 2Positive Advanced Breast Cancer: Final Results of NCIC CTG MA.31. 2015;33(14):1574-1583. doi:10.1200/JC0.2014.56.9590.

13. Neratinib after trastuzumab-based adjuvant therapy in patients with HER2-positive breast cancer (ExteNET): a multicentre, randomised, double-blind, placebo-controlled, phase 3 trial. 2016;17(3):367-377. doi:10.1016/S1470-2045(15)00551-3.

14. Slamon D, Eiermann W, Robert N, et al. Adjuvant Trastuzumab in HER2-Positive Breast Cancer. N Engl J Med. 2011;365(14):1273-83. doi:10.1056/NEJMoa0910383.

15. Guo L, Yuan P, Zhang J, et al. Analysis of molecular subtypes for the increased HER2 equivocal cases caused by application of the updated 2013 ASCO/CAP HER2 testing guidelines in breast cancer. Breast Cancer Res Treat. 2017;166(1):77-84.

16. Tong $Y$, Chen $X$, Fei $X$, et al. Can breast cancer patients with HER2 dual-equivocal tumours be managed as HER2-negative disease? Eur J Cancer. 2018;89:9-18. doi:10.1016/j.ejca.2017.10.033.

17. Sneige N, Hess KR, Multani AS, Gong Y, Ibrahim NK. Prognostic significance of equivocal human epidermal growth factor receptor 2 results and clinical utility of alternative chromosome 17 genes in patients with invasive breast cancer: A cohort study. Cancer. 2017;123(7):1115-23. doi:10.1002/cncr.30460. 
18. HER2 Gene Amplification Testing by Fluorescent In Situ Hybridization (FISH). Comparison of the ASCO-College of American Pathologists Guidelines With FISH Scores Used for Enrollment in Breast Cancer International Research Group Clinical Trials. 2016;34(29):3518-3528. doi:10.1200/JC0.2016.66.6693.

19. Vanden Bempt I, Van Loo P, Drijkoningen M, et al. Polysomy 17 in breast cancer: clinicopathologic significance and impact on HER-2 testing. J Clin Oncol. 2008;26(30):4869-74. doi:10.1200/JC0.2007.13.4296.

20. Does chromosome 17 centromere copy number predict polysomy in breast cancer? A fluorescence in situ hybridization and microarray-based CGH analysis. 2009;219(1):16-24. doi:10.1002/path.2574.

21. Clinical validation of. an array CGH test for HER2 status in breast cancer reveals that polysomy 17 is a rare event. 2009;22(9):1169-1175. doi:10.1038/modpathol.2009.78.

\section{Figures}



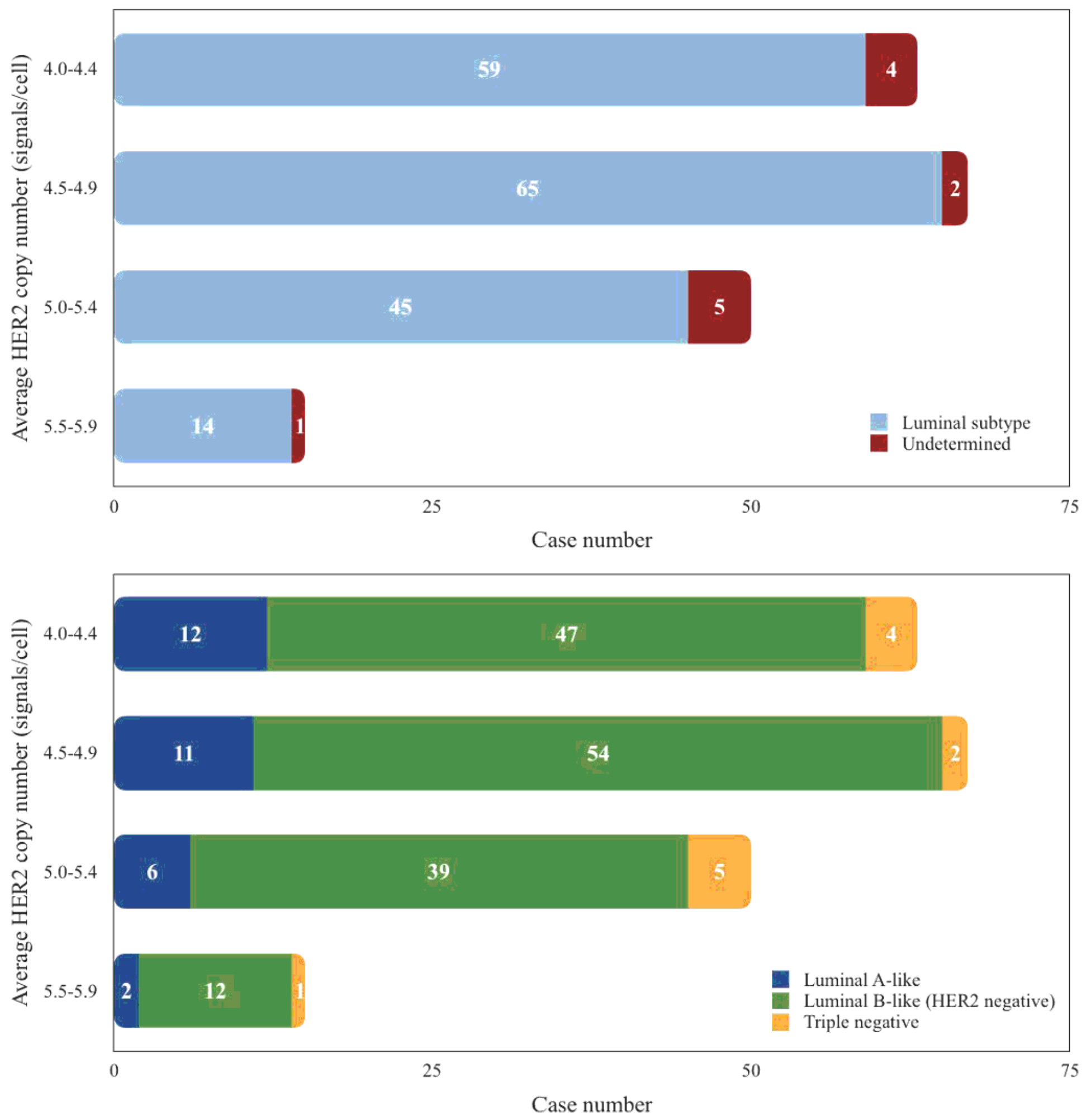

\section{Figure 1}

Molecular subtype distribution of HER2 FISH-equivocal breast cancers in terms of average HER2 copy number according to 2013 (A) and 2018 (B) ASCO/CAP guideline. 